\title{
Does airway integrated nasal packing after septal surgery improve body oxygenation?*
}

\author{
Shiou-Shyan Yu', Kai-Di Mei', Yung-Song Lin',2 \\ 'Department of Otolaryngology, Chi Mei Medical Center, Tainan, Taiwan \\ ${ }^{2}$ Department of Otolaryngology, School of Medicine, Taipei Medical University, Taipei, Taiwan
}

Rhinology 51: 243-248, 2013

DOl:10.4193/Rhino12.139

*Received for publication:

August 23, 2012

Accepted: May 12, 2013

\section{Summary}

Background: Airway integrated nasal packing reportedly improves body oxygenation after septal surgery. This randomized controlled study examined the effect of airway integrated nasal packing on oxygen saturation.

Methods: Eighty patients with septal deviation and hypertrophic turbinate were randomly divided into two groups: Group 1 patients were postoperatively packed with airway integrated Nasopore, and Group 2 patients were postoperatively packed with Nasopore without airway integration. The haemodynamic parameters and $\mathrm{SpO}_{2}$ (oxyhemoglobin saturation levels when measured using pulse oximetry) were sequentially checked. Nasal pain sensations were recorded using a visual analog scale.

Results: $\mathrm{SpO}_{2}$ was not significantly different between Groups 1 and 2. Nasal pain levels were significantly higher in Group 1 than in Group 2 at both $4(p=0.034)$ and $6(p=0.001)$ hours postoperatively. There were no significant differences between the two groups in the incidences of septal haematoma, perforation, or bleeding, or in sequentially checked heart rate, mean blood pressure, or respiration rate.

Conclusion: It was not evident that integrated airways improved the reduction of $\mathrm{SpO}_{2}$. However, Group 1 patients, with integrated airways, had more pain than did Group 2 patients, without integrated airways.

Evidence level: $1 \mathrm{~b}$.

Key words: nasal packing, nasal airway, septoplasty, oxygen saturation, sleep apnea, postoperative pain, septal haematoma

\section{Introduction}

Despite the general trend in rhinology to avoid nasal packing after sinus and septal surgery, packing materials are still used in the practice of otolaryngology, particularly after septal surgery for extreme deviation and in patients with a high risk of bleeding ${ }^{(1,2)}$. Nasal packing, however, is consistently associated with postoperative pain ${ }^{(3)}$. Additional potential complications include the worsening of obstructive sleep apnea ${ }^{(4,5)}$ and postoperative infections, which include toxic shock syndrome caused by post-septoplasty packing ${ }^{(6)}$.

Prior clinical studies ${ }^{(7-9)}$ on blood gas changes after nasal packing suggest that packing is frequently followed by hypoxia. Several animal studies have also examined the effects of arterial oxygenation on nasal packing. For example, significant depression of $\mathrm{PaO}_{2}$ (the partial pressure of oxygen in the blood) in the packed noses of unsedated dogs, but not in those of previously laryngectomized dogs, have been reported ${ }^{(10)}$, as well as significant hypoxia in lambs and ewes with a nasal blockade ${ }^{(9)}$. The 
$\mathrm{SaO}_{2}$ (arterial oxyhemoglobin saturation when measured using an arterial blood gas) levels of the lambs fell until their mouths opened, and then returned almost to control values. Another study ${ }^{(11)}$ reported a remarkable fall in $\mathrm{PaO}_{2}$ in rabbits (an obligate nasal breathing mammal) with bilateral nasal obstructions, although not in rabbits with only unilateral nasal obstructions. Based on the above studies, we hypothesized that providing an air passage inside nasal packing material would improve arterial oxygenation.

While significant reductions of $\mathrm{PaO}_{2}$ have been consistently reported in the literature, limits to the packing duration and to the alterations of packing materials have been advocated to limit nasal morbidity ${ }^{(12)}$. Moreover, two studies have examined the effects of packed airways on arterial hypoxia. One study ${ }^{(13)}$ found that the average $\mathrm{SpO}_{2}$ (arterial oxyhemoglobin saturation when measured using pulse oximetry) levels were significantly reduced in $33 \%$ of the patients that slept with nasal packing without an integrated airway, but were within normal limits for all patients with airway integrated nasal packing. Conversely, the other study ${ }^{(14)}$ found that airway integrated nasal packing produced no significant changes in the arterial $\mathrm{pO}_{2}$ or $\mathrm{O}_{4}$ saturation of patients given bilateral nasal packing ${ }^{(14)}$. It appears that airway integrated nasal packing has contradictory effects.

These studies raise two questions:

1. Does an integrated airway attenuate arterial hypoxia after nasal packing?

2. Do time differences affect arterial hypoxia?

To answer these questions, we did a randomized control study. An airway with a larger caliber might answer the first question. To investigate the significance of differences in check-up time, we also sequentially checked the $\mathrm{SpO}_{2}$ and hemodynamic parameters.

\section{Material and methods}

Participants

Eighty patients who underwent septoplasty between 2010 and 2011 for septal deviation and chronic hypertrophic rhinitis were enrolled in this study. Patients with the comorbidities of coronary heart disease, arrhythmia, or chronic obstructive lung diseases, or with a past history of any nasal surgery, were excluded. Preoperative examinations included chest and sinus X-rays, an electrocardiogram (EKG), a complete blood count, bleeding time measurement, and prothrombin time measurement. Block randomization was used for all patients to achieve balanced assignments in this prospective controlled study. The RV.UNIFORM $(0,1)$ function in SPSS for Windows (SPSS, Inc., Chicago, IL, USA) was used to generate random numbers and to assign distinct random permuted blocks to the patients. All participants were randomly divided into two groups: patients in Group 1 were

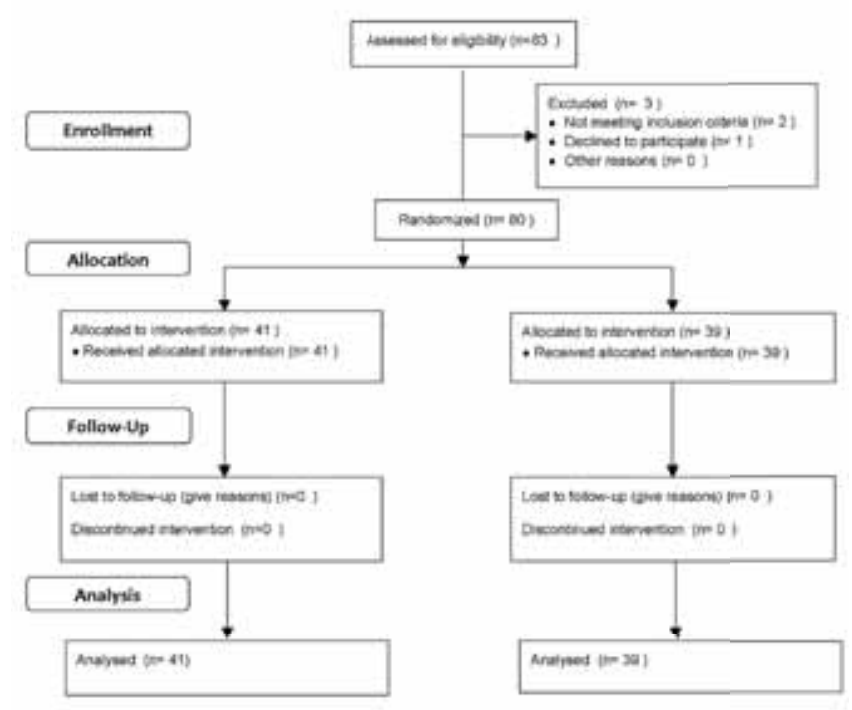

Figure 1. CONSORT flow diagram.

packed with airway integrated Nasopore (Polyganics, Groningen, the Netherlands), and patients in Group 2 were packed with Nasopore without airway integration. The surgeon did not know which type of nasal packing was to be used until the completion of the septal surgery (Figure 1). Each patient provided written consent before the study began.

\section{Surgery and nasal packing}

One surgeon performed all of the surgery. Local anesthesia (subcutaneously injected $2 \%$ lidocaine $\mathrm{HCl}$ ) was used for the surgery. The patients were premedicated with a combination of $3 \mathrm{mg}$ of intramuscularly injected morphine, $30 \mathrm{mg}$ of diphenhydramine, and $0.3 \mathrm{mg}$ of atropine 30 minutes before surgery. A swab culture of the nasal cavity was taken before surgery. Classical septoplasty was done with a Killian incision, and the warped cartilage and the bony hump or spur of the septal base was removed. A bilateral hemiturbinectomy was simultaneously performed for all patients. Electrocauterization was used to stop the bleeding from the trimmed turbinates. Continuous endoseptal sutures with 5-0 polyglycolic acid (Dexon; Covidien, Mansfield, MA, USA) were used from the bottom to the incision line to prevent septal haematoma and to secure the remaining septal cartilage and bones. Nasal packing was done with airway integrated Nasopore in Group 1 and Nasopore without airway integration in Group 2. For patients in Group 1, a silastic airway with an interior diameter of $5 \mathrm{~mm}$ was integrated into the soft nasal packing material. The opening of the nasal airway outside the nostril was funnel shaped at the rostral end to minimize crust clogging. The nasal airway length was extended $5 \mathrm{~mm}$ at the caudal end outside the nasal packing material. 
Preventive oral antibiotics (cephalexin monohydrate $250 \mathrm{mg}$, four times a day), along with oral antihistamines and decongestants (loratadine $5 \mathrm{mg}$ and pseudoephedrine $120 \mathrm{mg}$, twice a day) were given to each patient 30 minutes preoperatively and 48 hours postoperatively. To check $\mathrm{SpO}_{2}$, haemodynamic parameters, and the registration of pain on the pain scale, all of the participants were discharged on the 3rd postoperative day. Nasopore is autodegradable; therefore, it was unnecessary to remove the packing from the Group 2 patients. However, the silastic airways were removed on the third postoperative day from the noses of the Group 1 patients.

\section{Measurements}

The participants were asked to describe their pain using the visual analog scale (VAS) for pain ${ }^{(15-17)}$. The scores ranged from 0 to 10 , with 0 representing certainly not and 10 representing intolerable pain. The original time points were immediately preoperatively and 4,6 , and 12 hours postoperatively on the day of the operation, and 18 and 24 hours postoperatively on the day after the operation. The intramuscular injection of analgesics was prescribed pro re nata; however, there were no requests for analgesics within 6 hours after the operation. Based on a suggestion from the ethics committee, we did not check the VAS for pain between 9:00 p.m. and the next morning. Hence, not all evaluation points were checked, especially the evaluation points of VAS pain measurement at 12 hours post-surgery.

$\mathrm{SpO}_{2}$ was measured 30 minutes preoperatively with an $\mathrm{O}_{2}$ saturation monitor. Because these monitors did not significantly disturb the patients, we were able to record $\mathrm{SpO}_{2}$ levels sequentially at 4, 6, 12, 18, and 48 hours postoperatively.

Two months of follow-ups (with physical examinations and sinoscopy) were scheduled. Complications of postoperative bleeding, septal perforation, and septal hematoma were recorded. A follow-up bacterial culture was done on the 7 th postoperative day.

Table 1. Demographic characteristics of participants.

\begin{tabular}{|lccc|}
\hline & $\begin{array}{c}\text { Group 1 } \\
\text { (with airway) }\end{array}$ & $\begin{array}{c}\text { Group 2 } \\
\text { (without } \\
\text { airway) }\end{array}$ & p value \\
\hline Mean Age & 33.37 & 35.54 & 0.668 \\
\hline Sleep Apnea Syndrome & 7 & 6 & 0.329 \\
\hline GENDER & 28 & 19 & 0.078 \\
\hline Female & 13 & 20 & \\
\hline Male & 13 & & \\
\hline
\end{tabular}

\section{Data analysis}

A repeated-measures analysis of variance (ANOVA) test was used to judge the significance of the $\mathrm{SpO}_{2}$ reduction for each group. Independent t-tests were used to compare the continuous data between the two groups. Significance was set at $p<0.05$. Scores from the VAS for pain, which were measured preoperatively, served as the baseline to access the postoperative reduction of pain with no analgesics. The pain scores, $\mathrm{SpO}_{2}$ levels, and other hemodynamic parameters were measured 30 minutes preoperatively and served as references for calculating the reductions in those measurements.

The institutional ethics committee at Chi Mei Medical Center approved the protocol used in this study.

\section{Results}

Group 1 (with airway-integrated Nasopore) consisted of 41 patients, and Group 2 (without airway-integrated Nasopore) of 39 patients (Table 1). The average age at surgery was 34.43 years (range: 20-54 years). The gender distribution was 47 men and 33 women. No nasal packs were dislodged prematurely or removed in less than 48 hours. There was no significant bleeding upon the removal of the airway. All of the patients were discharged on the 3rd postoperative day. Two patients in Group 1 and three in Group 2 returned to the outpatient department for nasal bleeding between the 5 th and 7 th postoperative days. No repacking was needed. There was no septal haematoma, septal perforation, or postoperative infection.

There was no significant difference between the two groups in the pain scores taken 30 minutes preoperatively. A repeatedmeasures ANOVA showed significant $(p=0.006)$ differences in the Group 1 and Group 2 pain scores taken at the five checkpoint times. Group 1 patients expressed significantly higher pain scores at $4(p=0.034)$ and 6 hours $(p=0.001)$ post-surgery than did Group 2 patients.

There was no significant difference between the two groups in the $\mathrm{SpO}_{2}$ levels taken 30 minutes preoperatively (Table 2). Although a paired t-test indicated that the difference in checkpoint times affects arterial hypoxia (difference of the $\mathrm{SpO}_{2}$ levels between each checkpoint and the preoperative $\mathrm{SpO}_{2}$ level), a repeated-measures ANOVA test showed no significant difference in the $\mathrm{SpO}_{2}$ levels between the two groups.

Repeated-measures ANOVA also showed no significant differences between the two groups in the haemodynamic parameters: heart rate $(H R)$, respiration rate $(R R)$, and mean blood pressure (MBP) (data not shown).

Seven patients (17.1\%) in Group 1 and 6 patients (15.4\%) in Group 2 had histories of sleep apnea syndrome. In this subgroup, the differences in pain scores and $\mathrm{SpO}_{2}$ levels were not 
Table 2. Results of measurement for oxyhemoglobin saturation (arterial oxyhemoglobin saturation is called $\mathrm{SpO}_{2}$ when pulse oximetry is used to measure it) using pulse oximetry and the visual analog scale for pain.

$\begin{array}{cccc}\text { Group } 1 & \text { Group } 2 & \text { p-value } \\ \text { (with airway) } & \text { (without airway) } & \text { ANOVA }^{\mathrm{a}}\end{array}$

SpO2 $^{c}$ (followed by the number of evaluations at each time point)

$0.868^{*}$

Preoperative 98.63

(41)

(41)

(41)

(41)

(41)

(41)

98.15

0.02

(41)

(41)

(41)

(39)

(41)

(41)

$6 \mathrm{~h}$

$12 \mathrm{~h}$

$18 \mathrm{~h}$

$48 \mathrm{~h}$

0.52
3.31

2.00

1.53
98.74

(39)

0.726

97.77

97.36

97.28

97.41

98.10

0.00

(39)

(39)

$0.006^{*}$

(39)

Postoperative
2.62

2.62

1.99

1.32

0.96

0.52
(39)
0.333

0.453

0.562

0.245

0.643

0.872

$0.034^{*}$

$0.001^{*}$

0.051

0.079

0.982

${ }^{a}$ Independent t-test; ${ }^{b}$ repeated-measures analysis of variance; ${ }^{\mathrm{c}} \mathrm{SpO}_{2}$ is expressed in \%; ${ }^{\mathrm{d}}$ pain score visual analog scale is expressed in scores from 0 to 10 , all followed by the $p$-value of a paired t-test; ${ }^{*} p<0.05$.

significant between Group 1 and Group 2, nor were there any significant differences in the hemodynamic parameters.

On postoperative day 7, there were 10 patients (24.4\%) in Group 1 and 8 patients (20.5\%) in Group 2 who had developed positive bacterial cultures that were not found in the preoperative laboratory results; however, there was no significant difference in the incidence of a positive rate of growth of bacterial culture between the two groups. Finally, there were 2 patients (4.9\%) in Group 1 and 3 patients (7.7\%) in Group 2 with postoperative epistaxis, which was easily controlled using temporary packing with epinephrine-impregnated cotton pads on the bleeding sites. During the entire follow-up, no patient in either group had septal haematoma or septal perforation. To the end of the follow-up, there was no adhesion, nasal crusting, or mucosal atrophy detectable in a sinoscopic examination, nor was there any nasal deformity or saddle nose after surgery detectable during a physical examination.

\section{Discussion}

Septoplasty is one of the most common surgical procedures in the practice of otolaryngology. Nasal packs have been used in selected cases to reduce bleeding, prevent septal haematoma, and confine the residual septal cartilage and bones ${ }^{(18)}$. The universal use of nasal packing after septoplasty has not been supported in several studies. However, attempts have been made to reduce the morbidity of nasal packing in selected cases when nasal packing is necessary. Packing-related pain and arterial hypoxia are the most common morbidities.

\section{Postoperative pain}

To limit the compression caused by nasal packing, and to prevent an haematoma, a continuous endoseptal subperichondrial suture was used from the bottom to the incision.

There were significant postoperative differences in pain reduction between the two groups. The patients in Group 2, who were 
packed with Nasopore without an integrated airway, reported lower pain scores than did Group 1 patients, who were packed with airway integrated Nasopore. It appeared that the integrated airway reduced the softness of the packing material.

\section{Reduction of $\mathrm{SpO}_{2}$}

A comparison of the $\mathrm{SpO}_{2}$ during the six checkpoint times revealed no significant differences between Group 1 and Group 2. The integrated airway was initially designed to minimize arterial hypoxia. However, an integrated airway with an inside caliber of 5 $\mathrm{mm}$ yielded no difference in the reduction of $\mathrm{SpO}_{2}$ between the groups. Moreover, the haemodynamic parameters of HR, RR, and MBP, were not significantly different between the two groups. One of the discrepancies between the findings of Gupta et al. ${ }^{(13)}$ and Zayyan et al. ${ }^{(14)}$ may be related to the different time measurements of $\mathrm{SpO}_{2}$. Gupta et al. monitored $\mathrm{SpO}_{2}$ during sleep, particularly overnight. Zayyan et al., however, checked the blood gas before the operation and after the application of nasal packing. In the present study, we sequentially assessed $\mathrm{SpO}_{2}$ at the 4th, 6th, 12th, 18th, and 48th postoperative checkpoint hours. Attenuation of arterial hypoxia by using an integrated airway with nasal packing materials was not supported in the present study.

The underlying mechanism behind the nonsignificant difference in the levels of $\mathrm{SpO}_{2}$ between Group 1 and Group 2 remains unclear. We speculate two possible reasons. First, the average $\mathrm{SpO}_{2}$ level taken at the six time points ranged from $97.25 \%$ to 98.69\%. It was not difficult for patients with long-term nasal obstructions to adapt to breathing primarily through the mouth after surgery. Although the reduction in $\mathrm{SpO}_{2}$ might be statistically significant, this amount of reduction in $\mathrm{SpO}_{2}$ was clinically irrelevant for the patients in both groups. The ease of adapting to breathing primarily through the mouth after surgery may contribute to a lack of clinically relevant $\mathrm{SpO}_{2}$ differences between the two groups. Second, the softness and texture of the material might have affected $\mathrm{SpO}_{2}$ reduction in the present study. Nasopore has a different texture and degree of softness with and without integrated silastic airway tubes. We found that Group 1 patients reported higher pain scores than Group 2 pa- tients did. Considering the nasal mucosa compression and the stimulation related to the bronchomotor reflex ${ }^{(19,20)}$, the possible effects of a different texture and degree of softness from the packing materials may have diluted the effects of the integrated airway in this study. Moreover, it is possible for $5 \mathrm{~mm}$ of the integrated airway to be easily clogged by nasal crust or secretion. Additional observation and study are needed to explore the mechanism of whether (and why) the texture and softness of the packing material contributes to $\mathrm{SpO}_{2}$ reduction.

There was no significant difference in the proportion of patients with sleep apnea syndrome in both groups. For the small number [13] of patients with histories of sleep apnea syndrome, the $\mathrm{SpO}_{2}$, pain scores, and other haemodynamic parameters were not significantly different between Group 1 and Group 2 . However, because of the small number of patients with sleep apnea syndrome, we were unable to arrive at a conclusion about whether an integrated airway makes a significant difference in the $\mathrm{SpO}_{2}$, pain scale, and other haemodynamic parameters for this subgroup of patients.

\section{Conclusion}

Using integrated airways to improve the reduction of $\mathrm{SpO}_{2}$ saturation was not proved. However, patients with integrated airways had more pain than did patients without integrated airways. Differences in the packing material might be an important factor (among others) related to postoperative pain scores.

\section{Acknowledgement}

We thank Ms. Jin Lee from the Department of Medical Research for assisting with the statistical analysis. This research was funded by a grant from Chi Mei Medical Center.

\section{Authorship contribution}

SSY: clinical data assessment and recording, statistical analysis. KDM: data base recording, manuscript preparation.

YSL: study design, statistical analysis and manuscript writing.

\section{Conflict of interest}

None.

\section{References}

1. Acıoğlu E, Edizer DT, Yiğit O, Onur F, Alkan Z. Nasal septal packing: which one? Eur Arch Otorhinolaryngol. 2012; 269: 1777-1781.

2. Dubin MR, Pletcher SD. Postoperative packing after septoplasty: is it necessary? Otolaryngol Clin North Am. 2009; 42: 279285.

3. Nunez DA, Martin FW. An evaluation of post-operative packing in nasal septal surgery. Clin Otolaryngol. 1991; 16: 549-550.

4. Jensen PF, Kristensen S, Juul A, Johannessen
NW. Episodic nocturnal hypoxia and nasal packs. Clin Otolaryngol Allied Sci. 1991; 16: 433-435.

5. Regli A, von Ungern-Sternberg BS, Strobel WM, Pargger H, Welge-Luessen A, Reber A. The impact of postoperative nasal packing on sleep-disordered breathing and nocturnal oxygen saturation in patients with obstructive sleep apnea syndrome. Anesth Analg. 2006; 102: 615-620.

6. Toback J, Fayerman JW. Toxic shock syndrome following septorhinoplasty. Arch
Otolaryngol. 1983; 109: 627-629.

7. Yildirim A, Yasar M, Bebek Al, Canbay E, Kunt T. Nasal septal suture technique versus nasal packing after septoplasty. Am J Rhinol. 2005; 19: 599-602.

8. Ogretmenoglu O, Yilmaz T, Rahimi K, Aksöyek S. The effect on arterial blood gases and heart rate of bilateral nasal packing. Eur Arch Otorhinolaryngol. 2002; 259: 63-66.

9. Harding R, Buttress JA, Caddy DJ, Wood GA. Respiratory and upper airway responses to 
nasal obstruction in awake lambs and ewes. Respir Physiol. 1987; 68: 177-188.

10. Cavo JW, Kawamoto S, Berlin BP, Zollinger W, Ogura JH. Arterial blood gas changes following nasal packing in dogs. Laryngoscope. 1975; 85: 2055-2068.

11. Ramadan MF. Experimental nasal obstruction and changes in the arterial blood gases. Clin Otolaryngol Allied Sci. 1983; 8: 245-250.

12. Lubianca-Neto JF, Sant'anna GD, Mauri $M$, et al. Evaluation of time of nasal packing after nasal surgery: a randomized trial. Otolaryngol Head Neck Surg. 2000; 122 899-901.

13. Gupta M, Singh S, Chauhan B. Comparative study of complete nasal packing with and without airways. B-ENT. 2011; 7: 91-96.

14. Zayyan E, Bajin MD, Aytemir K, Yılmaz T. The effects on cardiac functions and arterial blood gases of totally occluding nasal packs and nasal packs with airway. Laryngoscope. 2010; 120: 2325-2330.

15. Ong KS, Seymour RA. Pain measurement in humans. Surgeon. 2004; 2: 15-27.

16. Coll AM, Ameen JR, Mead D. Postoperative pain assessment tools in day surgery: literature review. J Adv Nurs. 2004; 46: 124-133.

17. Williams AC. Outcome assessment in chronic non-cancer pain treatment. Acta Anaesthesiol Scand. 2001; 45: 1076-1079.

18. Ardehali MM, Bastaninejad S. Use of nasal packs and intranasal septal splints following septoplasty. Int J Oral Maxillofac Surg. 2009; 38: 1022-1024.

19. Sant'Ambrogio G, Widdicombe J. Reflexes from airway rapidly adapting receptors. Respir Physiol. 2001; 125: 33-45.

20. Fontanari $\mathrm{P}$, Burnet $\mathrm{H}$, Zattara-Hartmann MC, Badier M, Jammes Y. Changes in airway resistance induced by nasal or oral intermittent positive pressure ventilation in norma individuals. Eur Respir J. 1999; 13: 867-872.

Yung-Song Lin, MD

Department of Otolaryngology

School of Medicine

Taipei Medical University

250 Wu-Hsing Street

Taipei City 110

Taiwan

E-mail: kingear@gmail.com

\section{ERRATUM}

The following errors were made in the original article made by B. Lange: "Diagnosing chronic rhinosinusitis: comparing questionnaire-based and clinical-based diagnosis", which was published in Rhinology 2013; 51 (2): 128-136.

In the original article, the specificity for the original questionnaire was described as low. This was not correct. The specificity for the original questionnaire was high (93\%) and stayed at this level when using the new questionnaire. The error was of descriptive character and does not change the conclusion. A correction has been made in the main article and the abstract (an updated pdf file can be found online). Furthermore, Table 3 has been corrected. The overview of the number of persons with or without CRS according to the otolaryngologist and the questionnaire has been adjusted.

Table 3. Overview of number of persons with or without CRS according to the otolaryngologist and the questionnaire.

\begin{tabular}{|c|c|c|c|c|}
\hline \multirow{4}{*}{$\begin{array}{l}\text { Ques- } \\
\text { tion- } \\
\text { naire } \\
\text { based } \\
\text { CRS }\end{array}$} & & \multicolumn{2}{|c|}{ Clinical based CRS } & Total \\
\hline & & Yes & No & \\
\hline & Yes & $\begin{array}{c}45(34=76 \% \text { with } \\
\text { asthma or allergic } \\
\text { rhinitis) }\end{array}$ & $\begin{array}{c}19 *(16=84 \% \text { with } \\
\text { asthma or allergic } \\
\text { rhinitis })\end{array}$ & 64 \\
\hline & No & $\begin{array}{c}46(33=72 \% \text { with } \\
\text { asthma or allergic } \\
\text { rhinitis) }\end{array}$ & $\begin{array}{l}252(126=50 \% \\
\text { with asthma or } \\
\text { allergic rhinitis })\end{array}$ & 298 \\
\hline Total & & 91 & 271 & 362 \\
\hline
\end{tabular}

*According to the otolaryngologist these persons were diagnosed as: Normal (=7), chronic rhinitis $(=7)$, common cold $(=5)$, septal deviation $(=3)$.
This erratum shows the correct results section of the summary.

\section{Summary}

Results: Mean age of respondents to the postal questionnaire was 45.3 years and $52.9 \%$ were female. Persons with asthma were $8.4 \%$ and $26.2 \%$ were actual smokers. There was moderate agreement between questionnaire-based and clinical-based CRS. Sensitivity was low comparing questionnaire-based CRS with clinical-based CRS. Incorporation of self reported CRS and medical history in diagnosing CRS by questionnaire increased increased the agreement and sensitivity while specificity stayed at a high level. 